\title{
NEGATIVE LYAPUNOV EXPONENTS FOR DISSIPATIVE SYSTEMS
}

\author{
William G. HOOVER \\ Department of Applied Science, University of California at Davis-Livermore \\ and Department of Physics, Lawrence Livermore National Laboratory, Livermore, CA 94550, USA
}

Carol G. TULL

National Magnetic Fusion Energy Computer Center, Lawrence Livermore National Laboratory, Livermore, CA 94550, USA

and

Harald A. POSCH

Institute for Experimental Physics, Boltzmanngasse 5, University of Vienna, Vienna A-1090, Austria

Received 12 May 1988; revised manuseript received 15 June 1988; accepted for publication 16 June 1988

Communicated by A.A. Maradudin

By analyzing time-reversed trajectories from irreversible dissipative systems, we effectively reverse the order and signs of all the Lyapunov exponents. This reversal makes it possible to obtain the most negative Lyapunov exponents relatively easily. We illustrate the validity of this idea by studying the Lorenz model of Rayleigh-Benard instability.

\section{Introduction}

The Lyapunov exponents [1] describe the exponential divergence or convergence of phase-space objects: one-dimensional lengths, two-dimensional areas, three-dimensional volumes, and so on. The largest Lyapunov exponent, $\lambda_{1}$, describes the timeaveraged separation rate of two neighboring trajectories separated by the length $\delta_{\mathrm{L}}$ :

$\dot{\delta}_{L}=\lambda_{1} \delta_{L}$.

Thus $\lambda_{1}$ measures the rate at which one-dimensional phase-space objects grow. The rate at which a two-dimensional area $\delta_{\mathrm{A}}$ (defined by three neighboring trajectories) diverges or converges requires an additional exponent $\lambda_{2}$,

$\dot{\delta}_{\mathrm{A}}=\left(\lambda_{1}+\lambda_{2}\right) \delta_{\mathrm{A}}$.

Likewise, the sum of the first $n$ Lyapunov exponents describes the divergence or convergence rate of an $n$ dimensional phase-space volume. Provided that at least one Lyapunov exponent is positive, so that neighboring trjaectories diverge, the phase-space motion is called "chaotic". Unstable chaotic motion, as detailed by the Lyapunov exponents, is the mechanism which underlies the irreversibility of the second law of thermodynamics $[2,3]$, and so has been studied intensively.

Benettin, Calgani, and Strelcyn pioneered the numerical calculation of Lyapunov exponents in 1976 [4]. They time-averaged the rate at which a "satellite" trajectory moves away from a "reference" trajectory. A more primitive approach had already been successfully applied to a two-dimensional many-body fluid by Stoddard and Ford [5]. The corresponding treatment of the complete spectrum was claborated by Shimada and Nagashima [6]. To validate their numerical technique Shimada and Nagashima studied the "Lorenz" equations [7],

$$
\begin{aligned}
& \dot{x}=\sigma(y-x), \\
& \dot{y}=r x-y-x z, \\
& \dot{z}=-b z+y x .
\end{aligned}
$$


The Lorenz example problem, treated below, furnishes a crude description of unstable fluid flow. The unstable fluid exhibits "Rayleigh-Bénard" instability. The Lorenz model (3) is a set of three coupled equations describing the Fourier analysis of the velocity and temperature in a fluid heated from below in a vertical gravitational field. Close to equilibrium the fluid is motionless, and temperature decreases linearly with height. For a sufficiently large top-tobottom temperature difference, vortices develop. The Lorenz variables $x, y$, and $z$ describe the clockwise speed of vortex rotation, the horizontal variation in temperature, and the fluctuating variation in vertical temperature, all truncated to the first nonvanishing Fourier component. The positive parameters $\sigma, r$, and $b$ correspond respectively to the fluid's Prandtl number, the Rayleigh number, and the aspect ratio of the vortices. Popular interest in these Lorentz equations (3) and the corresponding Lorenz attractor (see fig. 1) spawned "chaos" as a popular and legitimate field of study. In this sense chaos is the dynamical behavior of Lyapunov-unstable systems.

Very recently, borrowing ideas from nonequilibrium molecular dynamics [8], we pointed out that a continuous version of Benettin's rescaling idea allows the Lyapunov spectrum to be determined by a Lagrange-multiplier method [9]. The Lagrangemultiplier method is efficient for small systems and shows very directly that the Lyapunov spectrum changes sign for reversible equations of motion. By using both Benettin's classical method and the more elegant Lagrange-multiplier method, we established the relatively simple form of the Lyapunov spectra for realistic many-body systems, both at, and away from, equilibrium [3]. The simple nature of the spectra suggests that a fairly complete characterization can be obtained if the first few positive and negative Lyapunov exponents are known. Here we show how to calculate negative Lyapunov exponents by analyzing, in reversed time order, stored points, previously generated along a forward-time phase-space trajectory. These negative Lyapunov exponents are of particular interest away from equilibrium, where the (negative) exponent sum is directly related to the irreversible entropy production [2]. The method illustrated here for ordinary differential equations can just as well be applied to irreversible equations such as the Navier-Stokes equations or to time series de-
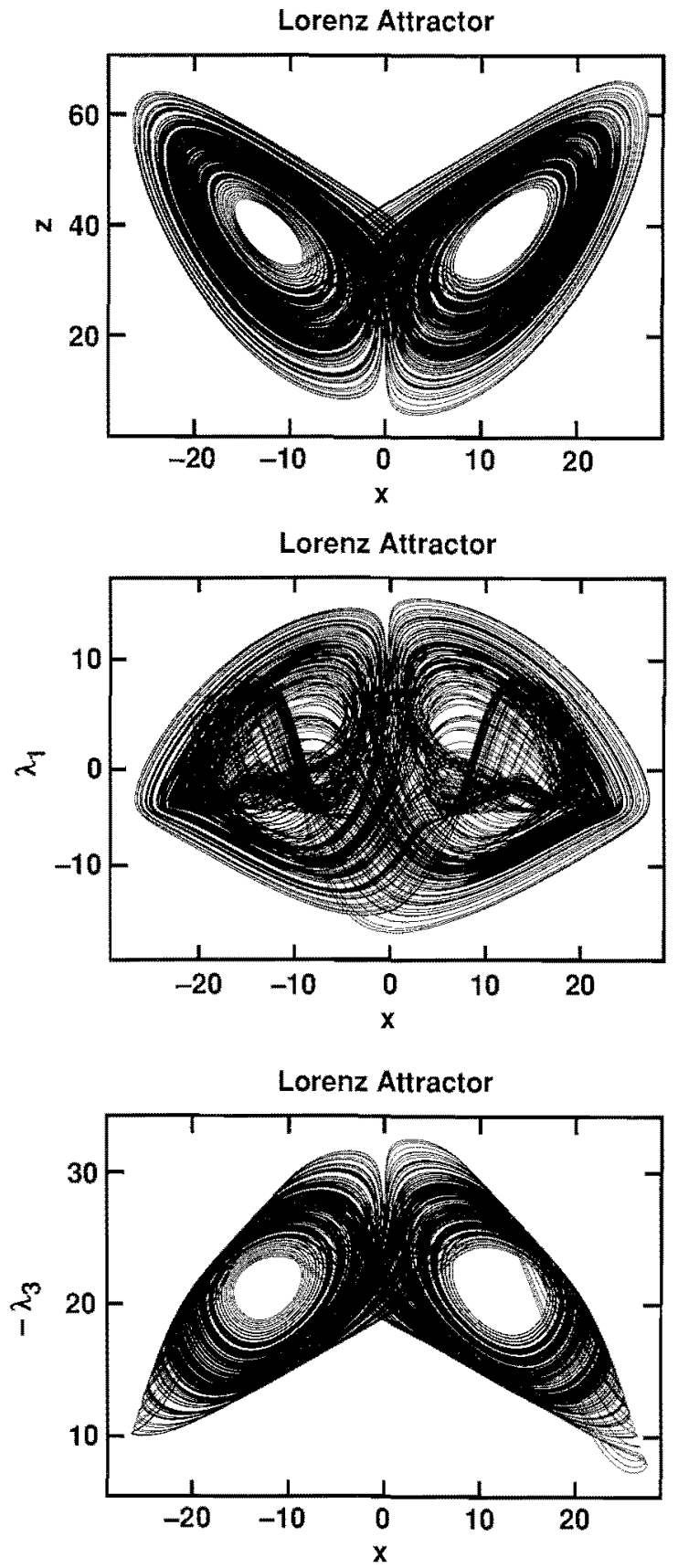

Fig. 1. Lorenz attractor trajectory corresponding to a time interval from 50 to 250 taken from a 300000-step run with timestep $\mathrm{d} t=0.001$ with the parameters listed in eq. (4) and initial point $(11,16,28)$. The Lorenz variable $z$ as well as the Lyapunov exponents $\lambda_{1}$ and $\lambda_{3}$ are plotted as functions of the Lorenz variable $x$. 


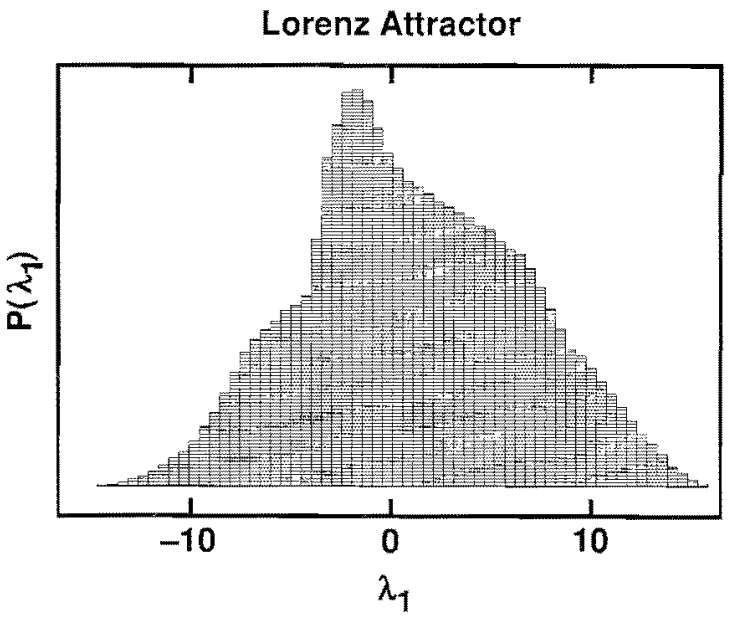

Lorenz Attractor

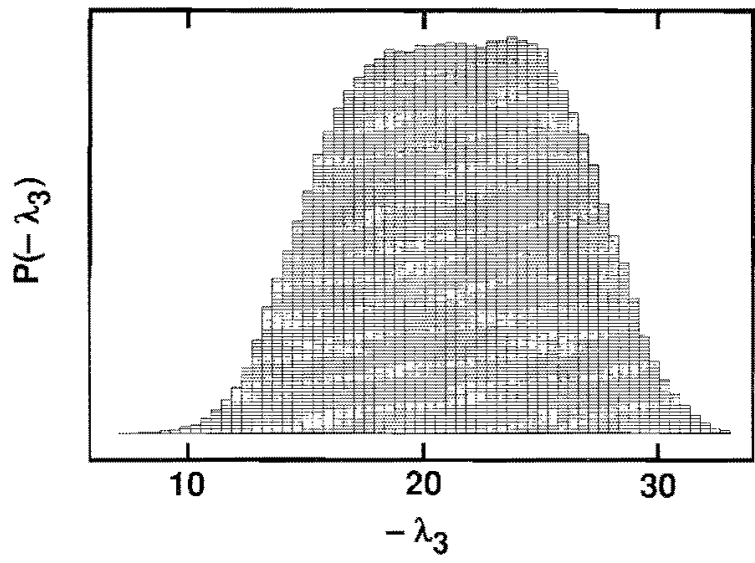

Fig. 2. Probability density for $\lambda_{1}$ and for $\lambda_{3}$ taken from the forward and backward versions of the attractor segment shown in fig. 1. The moments of the $\lambda$, distribution constitute the usual "spectrum of muitifractality".

rived from differential equations or experimental data. We illustrate the reversal idea here by applying it to the Lorenz model (3) with the same parameter choice studied by Shimada and Nagashima. The new method greatly reduces the time required to obtain negative Lyapunov exponents for systems with many degrees of freedom.

\section{Lorenz model calculations $[6,7]$}

In all of our calculations we use the parameter values from ref. [5] to describe an unstable RayleighBénard fluid in the chaotic regime:

$$
\begin{aligned}
& \dot{x}=16(y-x), \\
& \dot{y}=40 x-y-x z, \\
& \dot{z}=-4 z+x y .
\end{aligned}
$$

The largest Lyapunov exponent $\lambda_{1}$ is found by considering the motion of a "satellite" trajectory constrained to remain near a "reference" trajectory. We choose initial conditions near the attractor, shown in fig. 1 , at the point $(x, y, z)=(11,16,28)$. The direction of the satellite trajectory relative to the reference trajectory is specified by the vector $\delta=\left(\delta_{x}, \delta_{y}\right.$, $\delta_{z}$ ). The subsequent motion of the "unconstrained" displacement could then be calculated by solving the linearized equations of motion derived from (4):

$\dot{\delta}_{x \mathrm{u}}=16\left(\delta_{y}-\delta_{x}\right)$,

$\dot{\delta}_{y u}=40 \delta_{x}-\delta_{y}-x \delta_{z}-z \delta_{x}$,

$\dot{\delta}_{z \mathrm{u}}=-4 \delta_{z}+x \delta_{y}+y \delta_{x}$.

A Lagrange parameter $\lambda$, given by

$\lambda=\dot{\delta}_{u} \cdot \delta_{u} / \delta^{2}$,

when used in the new "constrained" equations of motion

$\dot{\delta}_{x \mathrm{c}}=\dot{\delta}_{x \mathrm{u}}-\lambda \delta_{x}$

$\dot{\delta}_{y \mathrm{c}}=\dot{\delta}_{\mathrm{yu}}-2 \delta_{y}$,

$\dot{\delta}_{z \mathrm{c}}=\dot{\delta}_{z \mathrm{u}}-\lambda \delta_{z}$

keeps the offset between the satellite and reference trajectory constant in time. The forward-time-averaged lagrangian multiplier $\langle\lambda\rangle_{\mathrm{f}}$ is the largest Lyapunov exponent $\lambda_{1}$. For small systems, this Lagrangemultiplier procedure is more efficient than Benettin's equivalent procedure of rescaling the vector $\delta_{u}=\left(\delta_{x}, \delta_{y}, \delta_{z}\right)$ at every timestep.

At a series of times equal to those chosen by Shimada and Nagashima, we recorded the time-averaged Lyapunov exponent in the forward direction of time, $\langle\lambda\rangle_{\mathrm{f}}=\lambda_{1}$. Then, the 409601 stored trajectory values of $x, y$, and $z$ were analyzed in reverse order. We show that the results are independent of the initial orientation of the reference-to-satellite vector $\delta$ by carrying out three separate calculations, with the initial direction of the vector $\delta$ chosen parallel to the $x, y$, and $z$ axes, respectively. We found that in the forward direction of time our largest Lyapunov ex- 
Table 1

The largest and smallest Lyapunoy exponents, $\lambda_{1}$ and $\lambda_{3}$, found using basis vectors initially oriented in the $x, y$, and $z$ directions as a function of time for the Lorenz model of eq. (4) of the text. The equations were solved with 409600 fourth-order Runge-Kutta timesteps of 0.01 and with initial conditions close to the attractor $(x, y, z)=(11,16,28)$. The entries $\tilde{\delta}_{x f} \cdot \delta_{y f}, \delta_{x f} \cdot \delta_{z f}$, and $\hat{\delta}_{y f} \cdot \delta_{z f}$ give the forwarddirection basis-vector dot products for the three different initial vectors, and indicate the rapid convergence of the satellite trajectory directions to the direction specified by the largest Lyapunov exponent, $\lambda_{1}$.

\begin{tabular}{|c|c|c|c|c|c|c|}
\hline Time & $\langle\lambda\rangle \times$ & $\langle\lambda\rangle_{\text {yf }}$ & $\langle A\rangle_{z f}$ & $\langle\lambda\rangle_{x b}$ & $\langle\lambda\rangle_{\text {: }}$ & $\langle A\rangle_{2 b}$ \\
\hline 2 & 0.669 & 1.293 & 1.553 & -22.302 & -21.930 & -21.960 \\
\hline 4 & 1,146 & 1.535 & 1.645 & -22.470 & -22.284 & -22.299 \\
\hline 8 & 1.323 & 1.518 & 1.573 & -22.453 & -22.360 & -22.368 \\
\hline 16 & 1.156 & 1.254 & 1.281 & -22.385 & -22.339 & -22.342 \\
\hline 32 & 1.348 & 1.397 & 1.410 & -22.404 & -22.380 & -22.382 \\
\hline 64 & 1.356 & 1.380 & 1.387 & -22.388 & -22.377 & -22.378 \\
\hline 128 & 1.361 & 1.373 & 1.376 & -22.381 & -22.375 & -22.376 \\
\hline 256 & 1.367 & 1.373 & 1.375 & -22.377 & -22.375 & -22.375 \\
\hline 512 & 1.375 & 1.378 & 1.379 & -22.376 & -22.374 & -22.374 \\
\hline 1024 & 1.377 & 1.379 & 1.379 & -22.376 & -22.375 & -22.375 \\
\hline 2048 & 1.378 & 1.378 & 1.378 & -22.377 & -22.376 & -22.377 \\
\hline 4096 & 1.377 & 1.378 & 1.378 & -22.377 & -22.377 & -22.377 \\
\hline ref. [5]: & & $1.37_{4}$ & & & $-22.37_{3}$ & \\
\hline Time & $\delta_{\lambda \vec{t}} \delta_{Y \mathrm{H}}$ & $\delta_{x \uparrow} \cdot \delta_{z t}$ & $\delta_{y f} \cdot \delta_{\exists}$ & & & \\
\hline 2 & -0.9906 & +0.9992 & -0.9992 & & & \\
\hline 4 & -1.0000 & +1.0000 & -1.0000 & & & \\
\hline
\end{tabular}

ponent, $\lambda_{1}$, agrees precisely with the ShimadaNagashima value, see table 1 . In the reversed time direction the largest apparent Lyapunov exponent becomes the negative of the most negative forwardtime exponent, $\langle\lambda\rangle_{\mathrm{r}}=-\lambda_{3}$. In this case also table 1 shows agreement with the Shimada-Nagashima results. It has since been emphasized that the directions corresponding to the Lyapunov exponents are also local variables [11]. We verified this property by showing that the final vector $\left(\delta_{x}, \delta_{y}, \delta_{z}\right)$ is independent of initial condition. Though we do not show the detailed results here, we have also verified numerically, using three orthonormal basis vectors,

Table 2

Coordinates and basis vector components in the forward and reversed directions at time 2048 at $(x, y, z)=(-7.030,-4.111$, $37.737)$.

$$
\begin{array}{lll}
\delta_{x:}=(+0.535, & +0.278, & -0.798) \\
\delta_{x f}=(-0.535, & -0.278, & +0.798) \\
\delta_{x f}=(+0.535, & +0.278, & -0.798) \\
\delta_{x b}=(+0.937, & -0.322, & +0.132) \\
\delta_{y b}=(-0.937, & +0.322, & -0.132) \\
\delta_{\mathrm{zb}}=(+0.937, & -0.322, & +0.132)
\end{array}
$$

that the entire spectrum of Lyapunov exponents, not just the largest one, is given correctly by analyzing the time-reversed trajectory. We have likewise verified the method for time-series data.

In table 2 we list the direction and location of these vectors at time 2048 going both forward and backward in time. These results are independent of the initial orientation of the vector $\delta=\left(\delta_{x}, \delta_{y}, \delta_{z}\right)$. It is noteworthy that there is no simple relationship between the orientations of the basis vectors in the forward and backward time directions for this dissipative system. In summary, we have shown that a reversed-time analysis of trajectory data (or timeseries data) can be used to obtain negative Lyapunov exponents as well as the positive ones.

The time savings can be considerable. Our recent 200000 timestep Lyapunov-spectrum simulations of three-dimensional 27-body shear flow required approximately $200 \mathrm{CRAY}-1$ hours each. The more than 25000 ordinary differential equations necessary to obtain the full spectrum can be reduced to a few hundred by using the stored-trajectory idea. That is, the trajectory data can first be processed forward, to 
find the first few positive exponents, and then backward, to find the most negative exponents.

\section{Discussion}

Our results demonstrate that negative Lyapunov exponents can be determined as quickly and easily as positive exponents, simply by analyzing trajectory data in reverse order. We emphasize that it is not necessary for the equations of motion to be time-reversal-invariant. Of course our procedure can be applied in the time-reversible case too. It is only required that the time derivatives can be calculated from the current state, so that either the future or the past can be generated. Even through the reversed trajectory has no physical significance, the replacement of contractions by expansions makes the negative Lyapunov exponents relatively easy to compute. In the many-body systems studied so far the Lyapunov spectra have a relatively simple shape, often a power law [3]. In such a case the first few positive and negative exponents are enough to characterize the complete distribution.

It is interesting to consider the situation in which data come from time series rather than analytic equations of motion [10-12]. In such a case the analog of equations of motion can be determined by considering the relative deformation of points on neighboring trajectories. Locally the deformation corresponding to an elastic strain-rate tensor, which describes the deformation of a phase-space hypersphere into a hyperellipsoid. Provided that sufficient data are available to construct deformation matrices analogous to eq. (5) of the text, the negative Lya- punov exponents can thus be obtained from time series.

\section{Acknowledgement}

This work was performed under the auspices of the United States Department of Energy under University of California - Lawrence Livermore National Laboratory Contract Number W-7405-Eng-48, and was further supported by the Austrian Fonds zur Förderung der wissenschaftlichen Forschung, Project P5455. Jim Viecelli and Jeff Kallman kindly provided both inspiration and assistance.

\section{References}

[1] H. Bai-Lin, Chaos (World Scientific, Singapore, 1986).

[2] B.L. Holian, W.G. Hoover and H.A. Posch, Phys. Rev, Lett. $59(1987) 10$.

[3] W.G. Hoover, H.A. Posch, and S. Bestiale, J. Chem. Phys. 87 (1987) 6665;

H.A. Posch and W.G. Hoover, Lyapunov instability of a dense Lennard-Jones fluid, Phys. Rev. A. (June 1988), to be published.

[4] G. Benettin, L. Galgani and J.M. Strelcyn, Phys. Rev. A 14 (1976) 2338.

[5] S.D. Stoddard and J. Ford, Phys. Rev, A 8 (1973) 1504.

[6] 1. Shimada and T. Nagashima, Prog. Theor. Phys. 61 (1979) 1605 .

[7] E.N. Lorenz, J. Atmos, Sci. 20 (1963) 130 .

[8] W.G. Hoover, Lecture notes in physics, Vol. 258. Molecular dynamics (Springer, Berlin, 1986).

[9] W.G. Hoover and H.A. Posch, Phys. Lett. A 113 (1985) 82; $123(1987) 227$

G.P. Morriss, Phys. Rev. A 37 (1988) 2118.

[10] J.M. Greene and J.-S. Kim, Physica D 24 (1987) 213.

[11] J.-P. Eckmann, S.O. Kamphorst, D. Ruelle and S. Ciliberto, Phys. Rev. A 34 (1986) 4971.

[12] A. Wolf, J.B. Swift, H.L. Swinney and J.A. Vastano, Physica D 16 (1985) 285. 